\title{
Intrinsic Fabry-Perot Sensors for Magnetic Field Detection
}

\author{
Christian Broadway, Frédéric Descamps, Damien Kinet, Christophe Caucheteur, and Patrice Mégret
}

\begin{abstract}
Within the context of ensuring stable nuclear fusion, it is important to monitor and control a number of parameters including the magnetic field associated with plasma circulation. Optical fibre sensing techniques have seen a surge in promulgation and research advances in recent years, due to their immunity to electromagnetic radiation and compact dimensions. Prior work has shown that fibre Bragg gratings are one method of recovering the induced magnetic field, with the main point of interest being their use as distributed point sensors. However, Bragg grating inscription leads to the creation of linear birefringence that increases detector noise and could obscure a given signal. We have hypothesised that by using an intrinsic Fabry-Perot cavity comprised of two identical Bragg gratings, we could obtain a more accurate detector with the removal of photo-induced birefringence in the detection region. We present a proof of concept optical fibre sensor based on an intrinsic Fabry-Perot cavity that shows spectrally visible amplitude modulation. Finally, we demonstrate faster data processing that allows real time monitoring of a given scenario.
\end{abstract}

Index Terms-Fiber Optic Current Sensors, Fiber Bragg grating sensors.

\section{INTRODUCTION}

$\mathbf{M}$ ANY applications exist for magnetic field sensors in the modern world, each with differing requirements for an eventual sensor. Three such examples are geophysical imaging, magnetic resonance imaging and field measurements within a TOKAMAK environment.

Magnetic sensing can be achieved by a range of optical and non-optical means. These include magneto-electric sensors[1], magneto-strictive devices [2], opto-fluidics [2], phased shifted Bragg structures [3], polarization based optical sensors [4], [5], [6] and optical birefringence analysis [7], [8]. Each methodology has specific advantages and disadvantages that can indicate specific applications.

Optical fibre sensors share an immunity to electrical interference and typically offer a passive sensor configuration. The wide variety of sensing configurations, materials, geometry and a typically small size can deliver sensitivity inversely proportional to its own dimensions has led to widespread research into applications from acoustics [9] to humidity [10].

One detection configuration is the inscription of periodic modulations within the core of a given optical fibre, known as a Bragg grating. These gratings cause a narrowband reflection around a central wavelength known as the Bragg wavelength.

C. Broadway, F. Descamps, D. Kinet, C. Caucheteur, and P. Mégret are with the Electromagnetism and Telecommunications Department of the University of Mons, 31 Boulevard Dolez, 7000 Mons, Belgium (email: christian.broadway@umons.ac.be, damien.kinet@umons.ac.be christophe.caucheteur@umons.ac.be, patrice.megret@umons.ac.be)
Bragg gratings are most commonly known for sensing temperature and strain [11] measurements but are also used for a wide variety of parameters including hydrogen detection [12] and acousto-optics [13]. Bragg gratings act as point detectors, isolating the reflected response from any perturbation along the fibre length. This has led to distributed sensing, where cascaded Bragg gratings can be used in applications such as civil engineering [14], providing a location for a given stress along with a given magnitude.

Working to develop point detectors for magnetic fields, our group has undertaken theoretical and practical work to apply Bragg grating technology in this domain [15], [16]. We have previously demonstrated a detector with good linearity that distinguishes between inherent birefringence in the optical fibre and externally induced birefringence, proving the concept and showing the potential for distributed magnetic field sensing. However, the inscription of Bragg gratings causes a significant localised increase in the inherent birefringence of an optical fibre within the grating region. This has rendered mandatory complex mathematical treatments to extract the induced field strength, presenting a blockage to real time analysis.

In this work, we present an intrinsic Fabry-Perot cavity composed of two identical Bragg gratings, inscribed within the fibre core and separated from each other by a given distance. This cavity is sensitive to perturbations between the gratings where no additional birefringence has been induced from the inscription process. We compare this cavity to a standard Bragg grating, demonstrating faster data extrapolation that allows for real time magnetic field monitoring.

\section{THEORETICAL PRINCIPLES}

The state of light polarization is the shape drawn by the tip of the electric field vector as a function of time at a given location along the fibre. For a monochromatic wave propagating in the $z$ direction of a Cartesian coordinate system $(x, y, z)$, the general solution of the wave equation can be stated as:

$$
\begin{aligned}
& E_{x}=E_{0 x} \cos \left(\omega t+\varphi_{x}\right) \\
& E_{y}=E_{0 y} \cos \left(\omega t+\varphi_{y}\right)
\end{aligned}
$$

where $E_{0 x(y)}$ are real numbers representing the amplitudes of the $x$ and $y$ components respectively with $\varphi_{x(y)}$ representing the given phase. Analysis of these two relations shows that the polarisation state is (1) linear when $\Delta \varphi=\varphi_{y}-\varphi_{x}=0$ or $\pi$, (2) circular when $E_{0 x}=E_{0 y}$ and $\Delta \varphi=\pi / 2$ or $3 \pi / 2$, and (3) elliptical in the general case. 
Equations (1) and (2) can be written compactly using the Jones vector, a 2 by 1 complex vector:

$$
\mathbf{V}(\omega)=\left[\begin{array}{ll}
E_{0 x} & e^{j \varphi_{x}} \\
E_{0 y} & e^{j \varphi_{y}}
\end{array}\right]
$$

Guiding properties of perfectly circular singlemode fibres leads to the propagation of two orthogonal degenerate modes $\mathrm{LP}_{01 x}$ and $\mathrm{LP}_{01 y}$, referred to as polarization modes or eigenmodes [17]. These modes propagate at the same velocity and experience the same effective refractive index $\left(n_{x}=n_{y}\right)$. However, optical fibres are always slightly elliptical and this imperfection removes the mode degeneracy, i.e. $n_{x}$ is no longer equal to $n_{y}$. The fibre therefore presents linear internal birefringence defined as:

$$
B_{l}=\left|n_{x}-n_{y}\right|
$$

Intrinsic birefringence in standard fibres typically corresponds to a refractive index difference in the region of $10^{-7}$. However, linear birefringence can also be externally introduced by mechanical perturbations such as stress.

For chiral materials, eigenmodes are the left-handed and right-handed circularly polarised fields, characterised by effective refractive indices $n_{L}$ and $n_{R}$. In a similar way to linear birefringence, circular birefringence can be defined as:

$$
B_{c}=\left|n_{L}-n_{R}\right|
$$

Under normal conditions, optical fibres do not present intrinsic circular birefringence, but twisting or magnetic fields can induce such birefringence.

When optical fibres are subjected to a longitudinal magnetic field $B$, an induced circular birefringence is created, this being known as the Faraday effect [18]. If polarised light is injected at the input of a fibre with length $l$, the output polarisation direction is rotated by angle $\alpha$ :

$$
\alpha=V B l
$$

where $V$ is the Verdet constant. A magnetic field can be retrieved by either examining the angle of rotation or the induced circular birefringence that causes the rotation.

One method to extract the induced birefringence is to work with the fundamental fibre parameters, such as polarization dependent loss (PDL) and polarization mode dispersion (PMD). The PDL is the ratio of the maximum transmitted power to the minimum transmitted power over all polarization states, while the PMD (also called differential group delay (DGD)) is the maximum difference in group delay over all polarization states.

Propagation within an optical fibre can be described by the Jones' formalism that relates $\mathbf{V}_{\text {out }}$ with $\mathbf{V}_{\text {in }}$ Jones vectors through a 2 by 2 complex transfer matrix:

$$
\mathbf{V}_{\text {out }}(\omega)=\left[\begin{array}{ll}
A(\omega) & B(\omega) \\
C(\omega) & D(\omega)
\end{array}\right] \mathbf{V}_{\text {in }}(\omega)
$$

It can be shown that PDL [19] and PMD [20] can be extracted from the measurement of the 4 coefficients $A(\omega)$, $B(\omega), C(\omega)$ and $D(\omega)$ of the transfer matrix.
We affirm that a given magnetic field will induce additional birefringence in an optical fibre and modify the aforementioned fibre properties, directly correlating changes in these values to the magnetic field. One method of measuring a magnetic field can be to observe these values.

\section{EXPERIMENTAL SETUP}

Fibre Bragg grating (FBG) based optical sensors can be used to measure magnetic fields as described in II. These FBGs are a periodic modulation of the fibres refractive index, creating a passband in the reflected spectrum from the fibre and a stopband in the transmitted spectrum. While FBGs are widely used in different sensing applications, the photo-inscription process that causes the refractive index modulation also causes an increase in the region of one order of magnitude in linear fibre birefringence. This obscures the induced birefringence and has previously required complex data processing, preventing real time monitoring [8].

To overcome this limitation, we have defined and inscribed an intrinsic Fabry-Perot cavity formed from two identical uniform FBGs of $2 \mathrm{~mm}$ long and separated by a cavity with a length of $6.83 \mathrm{~mm}$ as shown in figure 1 .

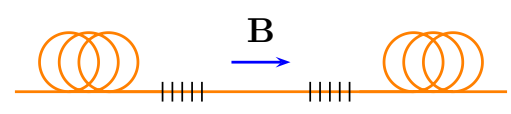

Fig. 1. Fabry-Perot cavity composed of two identical FBG with a cavity length of $6.83 \mathrm{~mm}$.

The Fabry-Perot cavity is placed inside a cylindrical electromagnet with an air gap of $6 \mathrm{~mm}$ (cf. figure 2). The cavity length of $6.83 \mathrm{~mm}$ was defined so as to be slightly larger than the air gap to isolate the FBGs from the magnetic field and only expose the cavity between electromagnet plates. The electromagnet generates a magnetic field when stimulated by a current source and a optical vector analyser from LUNA is used to retrieve the spectra, PDL and PMD from the transfer matrix (7).

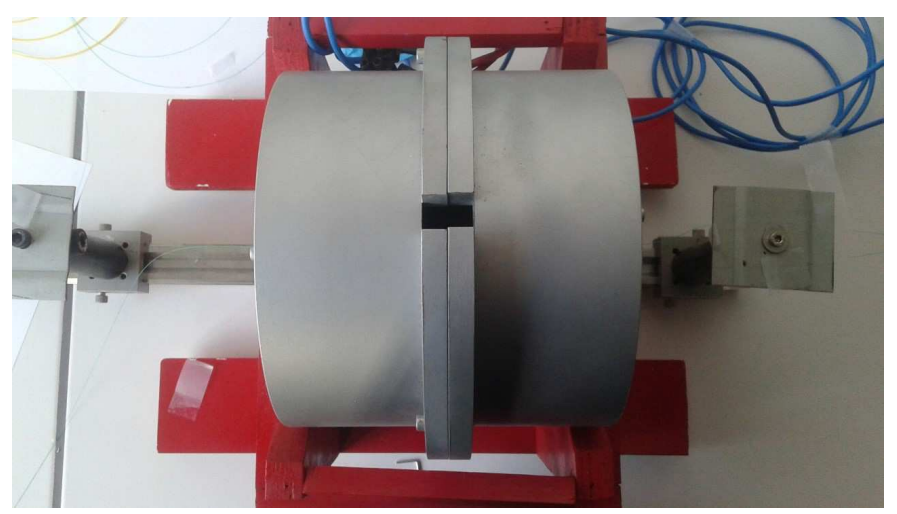

Fig. 2. Experimental setup: an electromagnet is used to generate a magnetic field between the two FBGs of figure 1.

We have characterized the electromagnet response by inducing a given current and measuring the induced magnetic field and change in internal temperature, the origin of which 
being electromagnet heating from the induced current. We observe in figure 3 a near linear relationship between current and magnetic field and a variation of temperature of less than $1{ }^{\circ} \mathrm{C}$.

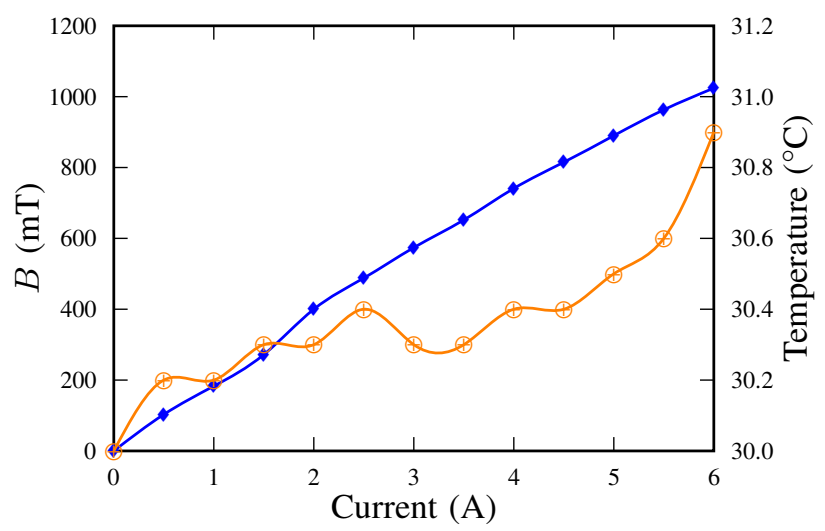

Fig. 3. Induced magnetic field strength (blue) and internal electromagnet temperature (orange) vs. current.

The Fabry-Perot cavity effect generates an interferometric peak pattern within the envelope of a uniform FBG spectrum (as shown in figure 4). There is a red-shift of the interference

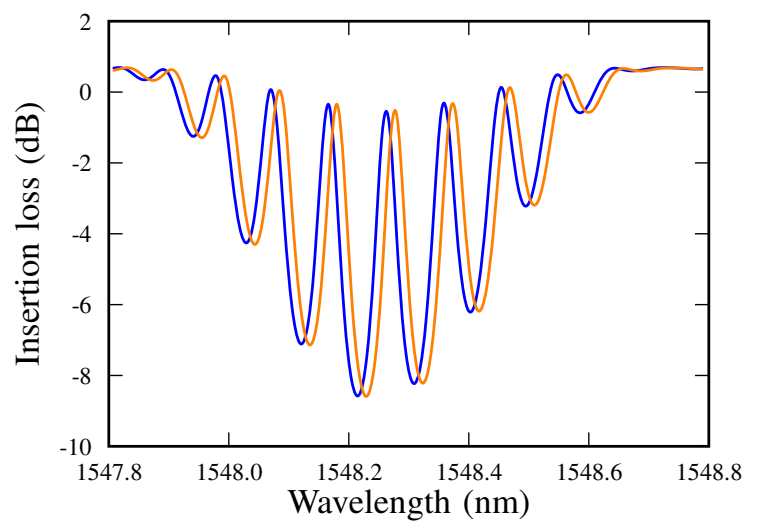

Fig. 4. Fabry-Perot spectral profile for currents of $0 \mathrm{~A}$ (blue) to $6 \mathrm{~A}$ (orange).

pattern between the two curves, which can be attributed to the associated temperature change, given that Bragg wavelength shifts in the region of $10 \mathrm{pm} /{ }^{\circ} \mathrm{C}$ have been well reported.

\section{Results}

Using the LUNA polarimeter, we obtain key propagation parameters along the fibre transmission line. As opposed to examining Mueller matrix coefficients as previously done [8], we take the fundamental properties of the fibre such as polarization dependent loss (PDL) and polarisation mode dispersion (PMD), analysing the results to determine a correlation to the induced magnetic field.

\section{A. PDL results}

As explained in section II, the PDL is based on the amplitude component of the cavity response. The induced modulation caused by the magnetic field can be observed in

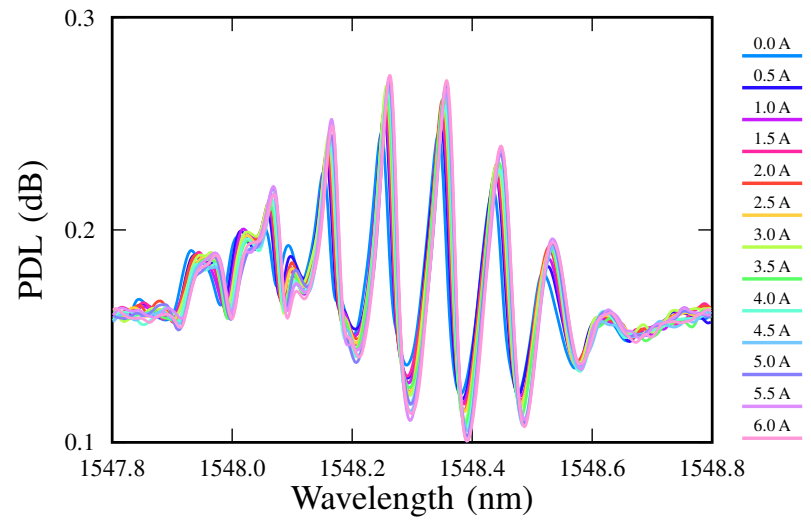

Fig. 5. PDL vs. wavelength per step current.

figure 5, showing the PDL spectrum change per 0.5 ampere step.

Due to the inhomogeneous nature of the profile, we identify the strongest central peak for data processing, giving figure 6 . The closer look afforded shows a distinctive wavelength shift along with a noticeable amplitude shift. As mentioned previously, a temperature change is responsible for the wavelength shift and originates in electromagnet heating.

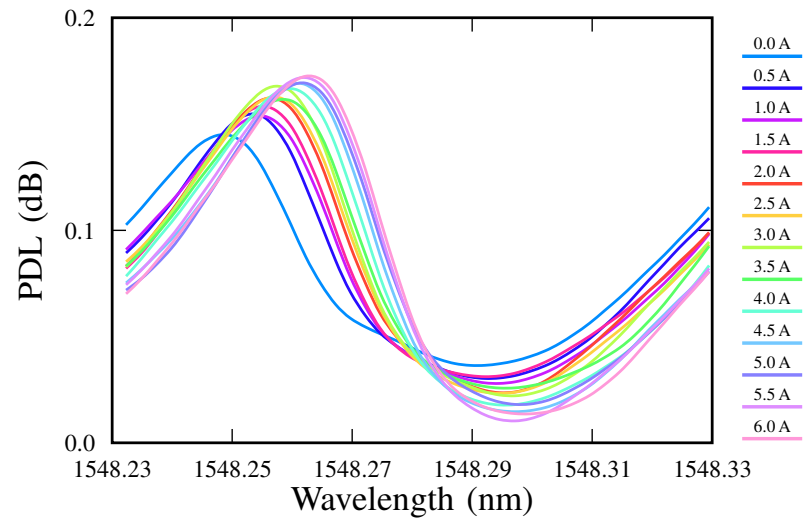

Fig. 6. Selected peak from figure 5.

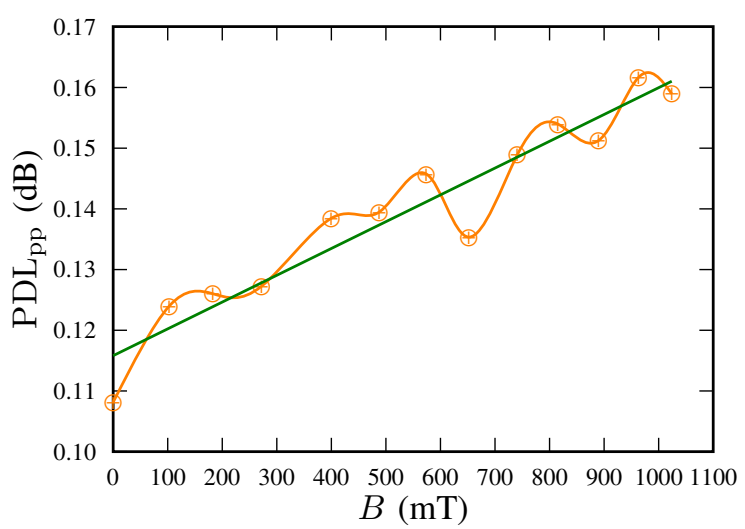

Fig. 7. Peak to peak PDL (orange) and least square fit (green) vs. the induced magnetic field.

From this, we calculate the peak to peak values for each ampere step, converting amperes to tesla as per figure 3, 
yielding figure 7 . We have displayed a least square fit, which can be expressed as:

$$
\mathrm{PDL}[\mathrm{dB}]=4.41277 \times 10^{-5} B[\mathrm{mT}]+0.115796
$$

We can observe an increase of $0.044 \mathrm{~dB} / \mathrm{T}$, where the precision of the LUNA is $\pm 0.03 \mathrm{~dB}$. Due to the narrow difference between these figures, it is difficult to confirm the trend with a good degree of precision, except to say that the trend is positive and conforms to expectations founded on our previous work.

\section{B. PMD results}

We have shown in section II that the PMD is based on the phase component of the cavity response. The induced modulation caused by the magnetic field can be observed in figure 8, showing the PMD spectrum change per 0.5 ampere step.

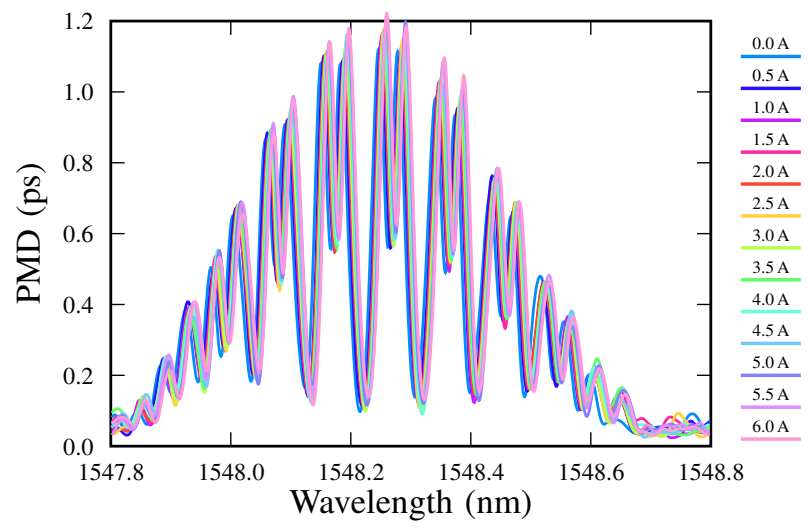

Fig. 8. PMD vs. wavelength per step current.

As with the PDL, we select the strongest and most homogeneous peak for data processing, seen in figure 9. Applying the same peak to peak value measurement, we correlate it to the induced magnetic field in figure 10 .

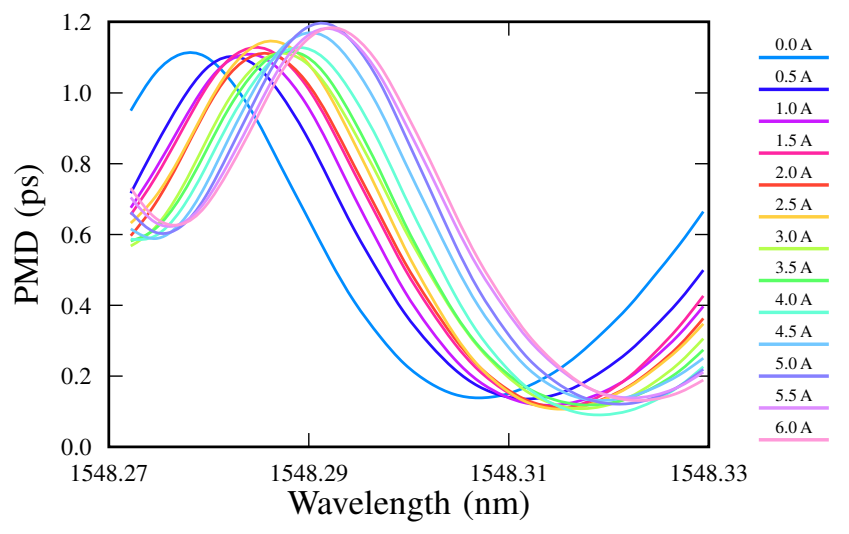

Fig. 9. Selected peak from figure 8.

A least square fit is again shown from which we give a derivation of the sensitivity:

$$
\mathrm{PMD}[\mathrm{ps}]=8.154 \times 10^{-5} B[\mathrm{mT}]+0.971917
$$

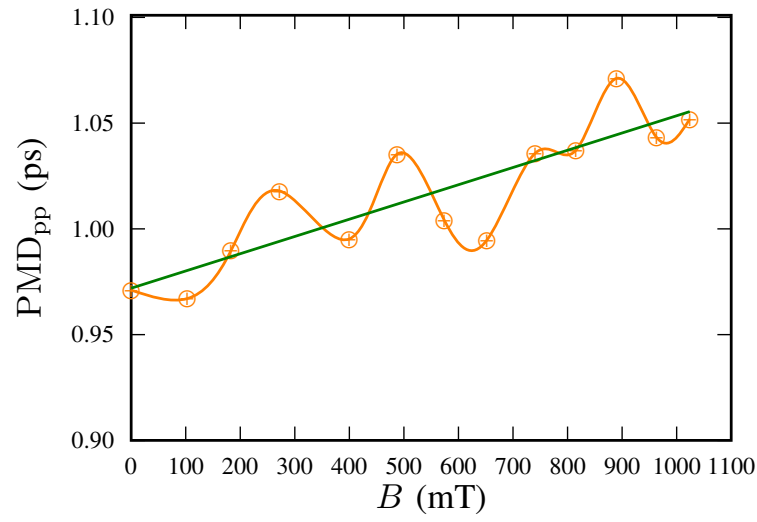

Fig. 10. Peak to peak PMD (orange) and least square fit (green) vs. induced magnetic field.

We can observe a sensitivity of $0.081 \mathrm{ps} / \mathrm{T}$ for a precision of $\pm 0.05 \mathrm{ps}$. While the trend of the response is positive, it is clear that the magnitude of the total change is of the same order as the precision. As with the PDL, we can see that the trend matches that observed in our previous work except that now the actual change is visible in the PMD spectrum without the need for further data processing. Additionally, a given peak of the PMD spectrum has a width of $60 \mathrm{pm}$, only yielding 24 points of actual data. This lack of sample density severely compromises the precision of the results. It is clear that were further studies to be based on the PMD, it would be better to have a polarimeter with a higher wavelength resolution.

\section{CONCLUSION}

We have presented an intrinsic Fabry-Perot cavity that can detect an induced magnetic field. The cavity exhibits no photoinduced birefringence and acts as a point detector, with the possibility for distributed sensing. We have demonstrated the possibility of real time monitoring by reducing the complexity of our data processing, made possible through the removed photo-induced birefringence. We have explored both phase and amplitude based detection and have demonstrated an amplitude component sensitivity of $0.044 \mathrm{~dB} / \mathrm{T}$ and a phase component sensitivity of $0.081 \mathrm{ps} / \mathrm{T}$.

We have successfully demonstrated the concept, but are conscious of the additional work to make this concept a more attractive one. Improvements include testing photonic crystal and larger diameter fibres, where greater fundamental sensitivity may be achievable. Changes to FBG inscription and an optimization of cavity parameters should deliver a more homogeneous spectrum that would permit data redundancy and an improved sensitivity. Finally, consideration of detector capabilities would yield more data points in the target region, giving a clearer response around peak values that could provide a more homogeneous progression.

\section{ACKNOWLEDGMENT}

C. Broadway is supported by H2020 European project Modern 2020, C. Caucheteur is supported by the F.R.S.-FNRS and P. Mégret is supported by the Belgian Science Policy IAP7/35. 


\section{REFERENCES}

11] T. Nan, Y Hui, M. Rinaldi, and N. Sun, "Self-biased $215 \mathrm{MHz}$ magnetoelectric NEMS resonator for ultra-sensitive DC magnetic field detection," Scientific reports, vol. 3, p. 1985, 2013. [Online]. Available: https://www.nature.com/articles/srep01985

[2] H. V. Thakur, S. M. Nalawade, S. Gupta, R. Kitture, and S. N. Kale, "Photonic crystal fiber injected with $\mathrm{Fe}_{3} \mathrm{O}_{4}$ nanofluid for magnetic field detection," Applied Physics Letters, vol. 99, p. 161101, 2011. [Online] Available: http://aip.scitation.org/doi/abs/10.1063/1.3651490

[3] P. Orr, P. Niewczas, M. Stevenson, and J. Canning, "Compound phaseshifted fiber Bragg structures as intrinsic magnetic field sensors," IEEE Journal of Lightwave Technlogy, vol. 28, no. 18, pp. 2667-2673, 2010.

[4] Y. Su, B. Zhang, Y. Zhu, and Y. Li, "Sensing circular birefringence by polarization-dependent parameters in fiber Bragg gratings and the influence of linear birefringence," Optical Fiber Technology, vol. 18, pp. 51-57, 2012.

[5] H. Peng, Y. Su, and Y. Li, "Evolution of polarization properties in circular birefringent fiber Bragg gratings and application for magnetic field sensing," Optical Fiber Technology, vol. 18, pp. 177-182, 2012.

[6] L. I. Chong-Zhen and W. U. Bao-Jian, "Theory and experiment on polarization-dependent loss of magnetooptical fiber Bragg grating system with a polarization controller," Microwave and Optical Technology Letters, vol. 53, no. 10, pp. 2224-2228, 2011

[7] S. Bette, C. Caucheteur, M. Wuilpart, and P. Mégret, "Theoretical and experimental study of differential group delay and polarization dependent loss of Bragg gratings written in birefringent fiber," Opt. Communication, vol. 269, pp. 331-337, 2007.

[8] F. Descamps, D. Kinet, S. Bette, and C. Caucheteu, "Magnetic field sensing using standard uniform FBGs," Optics Express, vol. 24, no. 23 pp. $26152-26160,2016$.

[9] C. Broadway, D. Gallego, A. Pospori, M. Zubel, D. J. Webb, K. Sugden, G. Carpintero, and H. Lamela, "A compact polymer optical fibre ultrasound detector," in Proc. SPIE 9708, Photons Plus Ultrasound: Imaging and Sensing, A. A. Oraevsky and L. V. Wang, Eds. vol. 9708. SPIE, 2016, pp. 970 813-970 813-7. [Online]. Available: http://dx.doi.org/10.1117/12.2212420

[10] W. Zhang and D. J. Webb, "Humidity responsivity of poly(methyl methacrylate)- based optical fiber Bragg grating sensors" Optic Letters, vol. 39, no. 10, pp. 3026-3029, 2014. [Online]. Available: http://dx.doi.org/10.1364/OL.39.003026
[11] C. Caucheteur, F. Lhommé, K. Chah, M. Blondel, and P. Mégret, "Simultaneous strain and temperature sensor based on the numerical reconstruction of polarization maintaining fiber Bragg gratings," Optics and Lasers in Engineering, vol. 44, no. 5, pp. 411-422, 2006.

[12] C. Caucheteur, M. Debliquy, D. Lahem, and P. Mégret, "Hybrid fiber gratings coated with a catalytic sensitive layer for hydrogen sensing in air," Optics Express, vol. 16, no. 21, pp. 16854-16 859, 2008.

[13] C. A. F. Marques, L. Bilro, L. Kahn, R. A. Oliveira, D. J. Webb, and R. N. Nogueira, "Acousto-optic effect in microstructured polymer fiber bragg gratings: Simulation and experimental overview," Journal of Lightwave Technology, vol. 31, no. 10, pp. 1551-1558, 2013.

[14] D. Kinet, P. Mégret, W. Goossen, L. Qiu, D. Heider, and C. Caucheteur, "Fiber Bragg grating sensors toward structural health monitoring in composite materials: Challenges and solutions," Sensors, vol. 14, no. 4 pp. 7394-7419, 2014.

[15] F. Descamps, M. Aerssens, A. Gusarov, P. Mégret, V. Massaut, and M. Wuilpart, "Simulation of vibration-induced effect on plasma current measurement using a fiber optic current sensor," Optics Express, vol. 22 no. 12 , pp. $14666-14680,2014$

[16] M. Wuilpart, M. Aerssens, A. Gusarov, P. Moreau, and P. Mégret, "Plasma current measurement in thermonuclear fusion reactors using a photon-counting POTDR," IEEE Photonics Technology Letters, vol. 29, no. 6 , pp. 547-550, 2017.

[17] M. Wuilpart and M. Tur, "Polarization effects in optical fibers," in Advanced Fiber Optics: Concepts and Technology, L. Thévenaz, Ed. EPFL Press, 2011, ch. 2, pp. 29-86.

[18] J. L. Cruz, M. V. Andres, and M. A. Hernandez, "Faraday effect in standard optical fibers: dispersion of the effective Verdet constant," Appl. Opt., vol. 35, no. 6, pp. 922-927, 1996.

[19] B. L. Heffner, "Deterministic, analytically complete measurement of polarization-dependent transmission through optical devices," IEEE Photonics Tech. Lett., vol. 4, pp. 451-454, 1992.

[20] — "Automated measurement of polarization mode dispersion using jones matrix eigenanalysis," IEEE Photonics Tech. Lett., vol. 4, pp. 1066-1069, 1992 